\title{
Macrophage Quantification in Different Breast Tumor Compartments
}

\author{
Anca Haisan ${ }^{1}$, T Petreus ${ }^{2}$, Daniela Jitaru ${ }^{3}$, M Danciu ${ }^{4}$ and E Carasevici ${ }^{3}$ \\ ${ }^{1}$ Emergency Medicine Department, “Gr.T. Popa” University of Medicine and Pharmacy, Iaşi, Romania \\ ${ }^{2}$ Department of Cell Biology, "Gr.T. Popa” University of Medicine and Pharmacy, Iaşi, Romania \\ ${ }^{3}$ Laboratory of Molecular Biology, Regional Institute of Oncology, Iaşi, Romania \\ ${ }^{4}$ Pathology Department "Gr.T. Popa" University of Medicine and Pharmacy, laşi, Romania
}

"Correspondence author: Anca Haisan, MD,Emergency Medicine Department, "St. Spiridon" Hospital, laşi, Romania, Independenţei Street, No 1, 700111, laşi, Romania, Tel: +40 (0) 2322177 81; Fax: +40 (0) 2322177 81; E-mail: anca.haisan@gmail.com

Received date: 2 December 2014, Accepted date: 30 December 2014, Published date: 9 May 2014

Copyright: (C) 2014 Haisan A, et al. This is an open-access article distributed under the terms of the Creative Commons Attribution License, which permits unrestricted use, distribution, and reproduction in any medium, provided the original author(s) and source are credited.

\begin{abstract}
Background and Aim: Breast cancer shows poor prognosis when tied with chronic inflammation mediated by tumor associated macrophages (TAM). However, TAM's multiple role, localization and prognosis are still debatable. Our study aims to quantify macrophage area and compare defined tumor morphological compartments: tumor associated stroma (TAS), tumor parenchyma and invasive front.

Material and Methods: Automated digital macrophage area quantification was standardized using a tissue cytometry system (TissueFAXS) on slides originated from 50 patients with invasive breast carcinoma. Macrophages were stained using immunohistochemistry with anti-CD68 marker. The three tumor compartments were manually delineated on the digital slides.

Results: Macrophage area was 3 times higher in lymph node-negative metastasis patients (pNO group) when compared to lymph node-positive group in tumor and invasive front compartments. Highly significant strong correlations were found between the three compartments only in pN0 group (i.e. $R>0.90, P<0.001$ ) while $p N+$ group showed weak ones suggesting various macrophage roles. CONCLUSION: Compartment-specific automatic quantification of macrophages in invasive breast carcinoma provides more insight on their role in tumor outcome. The new method applied in our study shows compartments' variability of local macrophage density and highlights behavior differentiated by the presence of metastasis.
\end{abstract}

Keywords: Breast Cancer; Tumor Compartments; Macrophage Area; Cd68; Invasive Front; Computer Image Analysis

\section{Introduction}

Many malignancies, including breast cancer, show poor prognosis when associated with chronic inflammation, usually mediated by special monocyte-derived cells - the tumor associated macrophages (TAM) [1-3]. In solid tumors, macrophages represent a constant presence. Due to their dual behavior, pro and anti-inflammatory [4-6], the TAM effects on tumor site depend on the macrophage secreted molecules [7-9] and also on their surface markers and density. CD68 TAMs were subject for wide investigations and reviews, mainly in breast cancer [10-14]. Mantovani et al demonstrated that TAMs are usually of M2 type, with enhanced tumor inducing functions, cell survival supportive effects and antiapoptotic [3,15-17]. CD68 TAMs distribution in breast cancers was investigated by various methods, mainly immunohistochemistry on tissue microarrays blocks $[14,18,19]$. Evaluation methods for CD68 TAMS in various tumor areas or according to lymph node involvement are also fluctuating, from microscopic observations to molecular biology methods [20-22]. Data interpretation regarding CD68 TAMs presence in breast tumors shows inconsistent correlations between TAMs localization and prognosis. Thus, the aim of this study is to perform a computer assisted measurement of relative stained area index in three defined tumor compartments - tumor associated stroma, tumor parenchyma and invasive front - in order to show differences in local density for CD68 tumor associated macrophages.

\section{Materials and Methods}

\section{Patients and tissues}

Our cohort was comprised of 50 samples of invasive breast carcinoma patients between 37 and 70 years old (mean age 57), of No Special Type (NST). Patients were without any adjuvant hormonal or chemotherapy previous to the surgical resection. We have obtained additional information for each patient, as medical pathological records; each tumor was classified according to its' pathological pattern.

\section{Immunhistochemistry}

All tumors were formalin fixed. Sections $5 \mu \mathrm{m}$ thick from paraffin embedded tumor blocks were mounted on adhesive slides for both test samples and negative controls. Sections were deparaffinated in xylene baths and rehydrated in graded alcohols to water $(90 \%, 70 \%, 50 \%$ and 30\%) 5 minutes each bath. For antigen retrieval we used HIER method with a steamer $\left(98^{\circ} \mathrm{C}\right.$ for 30 minutes), in high $\mathrm{pH}$ Dako retrieval solution; after 30 minutes, slides were cooled at room temperature in retrieval solution, then washed in Tris-buffered saline (TBS) solution 3 times for 5 minutes each. Endogenous peroxidase activity was 
quenched by $0.3 \mathrm{H} 2 \mathrm{O} 2$ at room temperature for 10 minutes. After TBS washing again (3 times for 3 minutes each), blocking solution was used (Protein Block Serum-Free, Ready to use, Dako). The slides were then washed with TBS 3 times of 3 minutes each. Test lot was incubated over night at 4 Celsius degrees after applying primary antibody mouse monoclonal ab955 [KP1] anti CD68 Abcam, at a 1:300 dilution. The negative control lot was incubated in the same manner but using monoclonal mouse IgG1 antibody instead of the primary antibody. Second day we applied on both lots biotinilated link and detection complex, LSAB-kit Dako. Developing reaction was made with DAB chromogen and after that, counterstained with Mayer's haematoxylin, fixed in tap water, dehydrated in alcohol and xylene clarified, mounted and cover slipped.

\section{Scanning procedure}

Digitization and analysis were performed with TissueFAXS 3.5 (TissueGnosticsGmbh, Vienna, Austria) which included the scanning system in addition to the cytometry analysis software (TissueQuest and HistoQuest). The scanner consisted of a Zeiss Imager Z2 microscope, a 3 Megapixel colour camera Pixelink PL-623 CF and a motorized stage from Maerzhauser with 8-slide insert for the batch scanning mode. The white light lamp was a VISLED based on LED technology providing a stable intensity during the study. The analysis computer was a HP Z400 equipped with an Intel Xeon W3565 processor at $3.2 \mathrm{GHz}$ running Windows7 (64bit).

A 10x objective was used for the image acquisition. Established microscope parameters were checked before scanning, as well as the Koehler illumination procedures described in Zeiss Imager manuals. The sensor of the camera was aligned to the axis of the stage so that the angle between them was less than 0.01 degrees, thus minimizing mosaic image alignment shifts. TissueFAXS 3.5 saved the images in JPEG format with a $95 \%$ quality index. The virtual slides were merged using a $15 \%$ image overlap between neighboring images. Shading compensation was performed using a shading correction image acquired with same camera settings. Exposure time of the camera was set such that pixels in white background had a value 230-240 on all red, green and blue channels. White balance was realized only once using the automatic feature in an image without tissue. Linear behavior of the sensor was ensured by disabling gamma adjustments. Lamp and camera parameters were saved in a TissueFAXS intensity profile, then reused for all slides of the study, such that images were acquired with a consistent comparable quality. The digital slides were 58 GB in size on a NTFS formatted hard disk; Total scanned area over the entire set of 50 patients was $46862 \mathrm{~mm}^{2}$.

\section{Definition of regions of interest}

Visual analysis of the digital slides was performed in an initial phase using contextual assessment from a pathological point of view. Locations of tumor area (parenchyma) (T), tumor associated stroma (TAS) and invasive front (IF) were identified in each section. The tumor area having an irregular shape, consisted in ducts, nests and cords of tumor cells. We considered the invasive front of the tumor the interface between the adjacent breast tissue and the periphery of tumor. The pattern of the tumor growth was often infiltrating and widely dissecting the normal breast tissue, without clear boundaries between host tissues and tumor. Tumor budding was found in the invasive front, exhibiting small aggregates or isolated tumor cells. Tumor associated stroma (TAS) was outlined as the fibro-vascular tissue (microenvironment) surrounding or including the malignant cells. Studies of the desmoplastic reaction found that TAS is a useful histological prognostic parameter in breast cancer [27]. Using Ueno's criteria [27] for classification of fibrotic cancer stroma we classified our cases as having: mature, intermediate or immature fibrotic stroma.

Several sites $(n=1-3)$ were chosen for analysis using Standard Regions of Interest (ROI) tool, each site selected with a predefined disk shape of $1 \mathrm{~mm}$ diameter. Contours of each area were highlighted in green, blue or red, for stroma (green), tumor (blue) or invasive front (red), respectively. Colored highlighting increased speed of secondary opinion analysis and during post-analysis checks since the areas were easily observed. Selections of ROIs were made avoiding folded tissue, areas with mechanical disrupted morphology generated by cutting, air bubbles within mounting medium or major staining artifacts.

ROIs were defined in two phases. First, 2 medical doctors independently highlighted the areas for analysis. A second review phase was performed by the entire team for reaching final agreement on ROI sites in all samples (Figure 1,2 and 3).

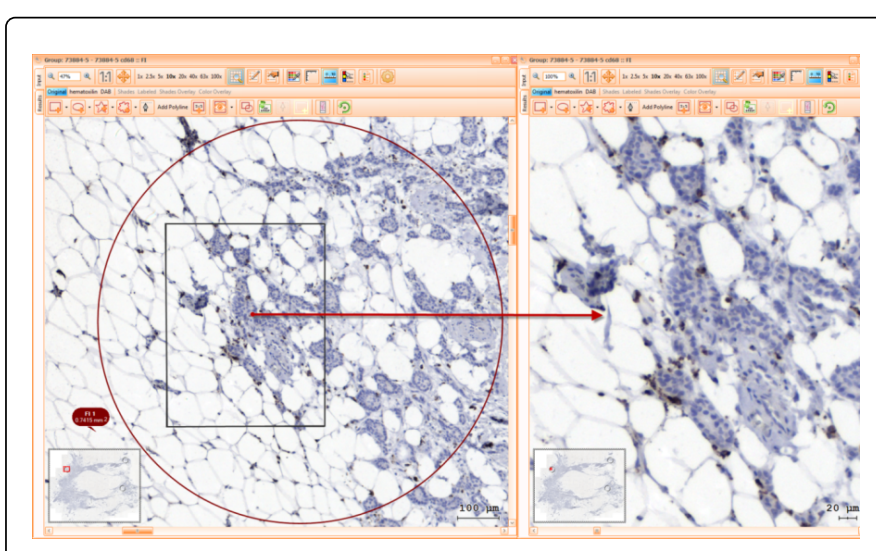

Figure 1: Selection of invasive front stained with CD68; detailed view on the right side.

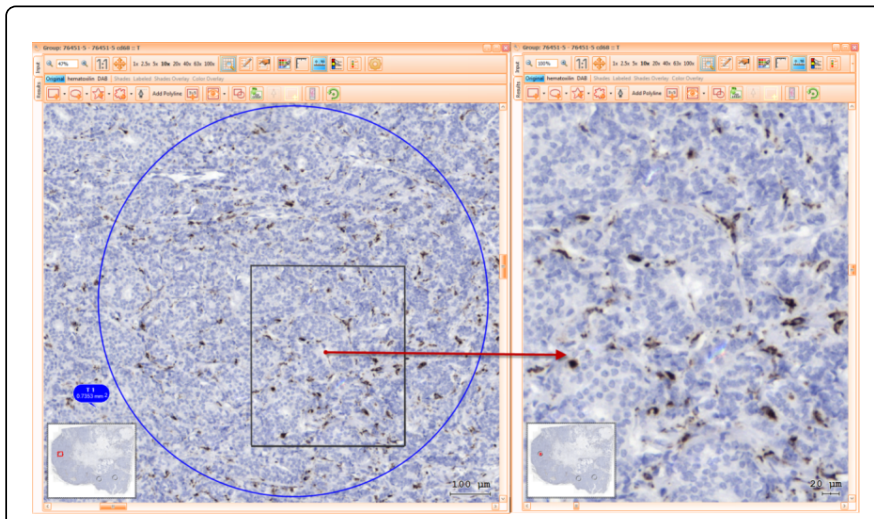

Figure 2: Selection of tumor stained with CD68; detailed view on the right side. 


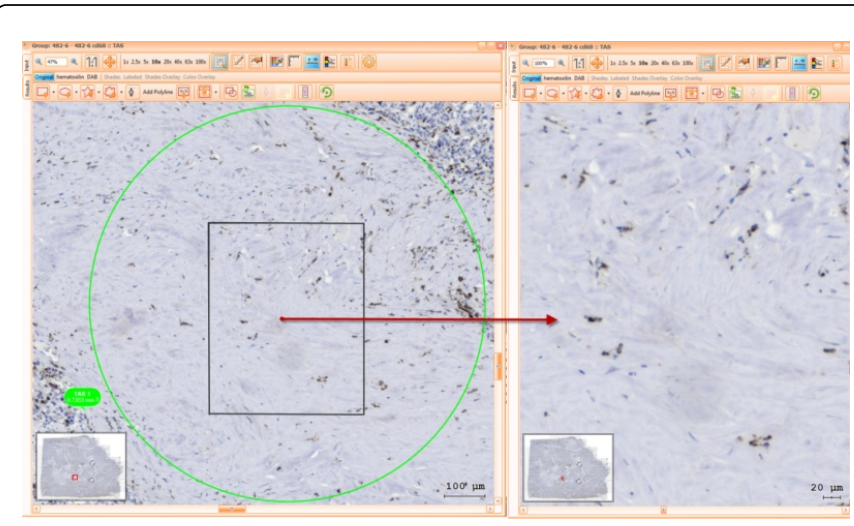

Figure 3: Selection of tumor associated stroma stained with CD68; detailed view on the right side.

\section{Measurement procedure}

HistoQuest 3.5 software was used for quantitation. HistoQuest analysis projects imported TissueFAXS digital slides, considering available shading correction and image tile overlapping information. The software unmixes the color RGB image into marker-specific optical density images with an algorithm for colour separation selected using Single Reference Shade module. Thus it separates Hematoxylin and CD68-DAB into their Optical Density (OD) counterparts. The training procedure is done by pointing with the mouse pixels for each of the two stains. In the CD68-DAB optical density images, positive areas were measured by manual thresholding and quantifying StainedArea (SA) using Total Area Measurement option. The threshold was found after an iterative search in several samples by looking at the resulting overlays of detected contours on the original images. The software allows interactive threshold definition. Best settings found on representative small test regions were saved in a "marker profile" of HistoQuest and then applied in all analysis projects of the study using the "batch analysis" module, for unattended automatic computation.

\section{Validation of macrophage area detection}

After quantification, a visual validation of proper SA identification was done on all digital slides. Each project was reopened for assessment of overlays of macrophage contours on the colour images. "Add event" from "Manual Correction Module" was used to indicate areas which were too weak for automatic detection. Identified areas which were not macrophages, such as staining artifacts or high background, were deleted from analysis using "Delete Events". Only 5 digital samples needed manual corrections of the automatic stained area identification.

\section{Extraction of quantitative data}

Validated measurement results were extracted from the analysis projects using Batch Export module in a single Excel file: total Analyzed Area (AA) and total Stained Area (SA) of each domain of each patient. Derived results were calculated directly in Excel: the Relative Stained Area (RSA) for each domain (RSA percent $=100 \mathrm{x}$ SA / AA). SPSS 19 software was used for statistical analysis.

\section{Results}

\section{Macrophage quantitation}

Macrophage stained area was measured in each compartment of TAS, $\mathrm{T}$ and IF, for patients in group $\mathrm{pN}_{0}$ (no metastasis), for those in pN positive (node metastasis present) as well as for all patients (Table $1)$.

Table I: Average values for TAS, T and IF compartments in N0 group, N+ group and all patients respectively. TAS Tumor Associated Stroma; T Tumor aria (parenchyma); IF Invasive Front.

\begin{tabular}{|l|l|l|l|}
\hline \multirow{2}{*}{ Group } & \multicolumn{3}{|c|}{ Average Relative Stained Area (RSA) } \\
\cline { 2 - 5 } & TAS - RSA & T - RSA & IF - RSA \\
\hline N0 & 0.46 & 0.63 & 0.58 \\
\hline N+ & 1.06 & 1.79 & 1.5 \\
\hline Overall & 0.97 & 1.62 & 1.37 \\
\hline
\end{tabular}

Patients in pN0 showed values of $0.46,0.63$ and 0.58 for TAS, T and IF respectively. Patients in group $\mathrm{pN}$ positive revealed average compartment measurements of $1.06,1.79$ and 1.5 respectively, values 2-3 times higher than those in $\mathrm{N}_{0}$ group. When looking at the entire population of patients, mean values were calculated as $0.97,1.62$ and 1.37. All three groups revealed the same sorted trend of higher CD68 areas in tumor, followed by lower values in invasive front; whereas tumor associated stroma had lowest values of all three compartments. Values from Table I were displayed graphically in Figure 4.

\section{Statistical analysis}

Pearson correlations between all possible pairs of compartments were calculated for patients without node metastasis (Table II).

Strong correlations were found in all pairs $(0.908,0.922$ and 0.749 respectively), with a highly significant $\mathrm{p}$ values (less than 0.05 for all cases). 


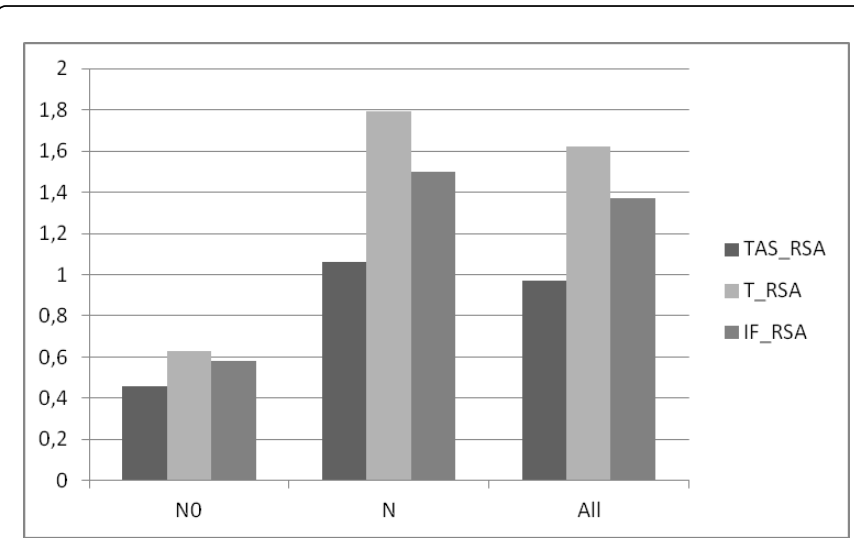

Figure 4: Graph showing average values of CD68-RSA measurements for $\mathrm{N} 0, \mathrm{~N}+$ and entire population in all three compartments (TAS, T and IF) TAS Tumor Associated Stroma; T Tumor aria (parenchyma); IF Invasive Front; RSA Relative Stained Area.

For patients with node metastasis, a relatively weak correlation was found only in the "TAS \& T" pair, but with a highly significant $p$ value (less than 0.001) (Table 3).
Table II: Statistical analysis for all compartment pairs for patients without node metastasis. TAS Tumor Associated Stroma; T Tumor aria (parenchyma); IF Invasive Front.

\begin{tabular}{|l|l|l|l|l|}
\hline \multirow{2}{*}{$\begin{array}{l}\text { Compartment } \\
\text { pairs }\end{array}$} & \multirow{n}{*}{$\mathbf{n}$} & \multicolumn{2}{|c|}{ Correlations } & $\begin{array}{l}\text { Paired } \\
\text { Differences }\end{array}$ \\
\cline { 3 - 5 } & & $\begin{array}{l}\text { Pearson } \\
\text { correlation (R) }\end{array}$ & $\mathbf{P}$ & $\mathbf{P}$ \\
\hline TAS \& T pair & 10 & 0.908 & $<0.001$ & 0.108 \\
\hline TAS \& IF pair & 10 & 0.922 & $<0.001$ & 0.204 \\
\hline T \& IF pair & 10 & 0.749 & 0.013 & 0.560 \\
\hline
\end{tabular}

Statistical assessment of entire patient cohort showed a significant ( $\mathrm{P}$ less than 0.001$)$ but weak correlation $(\mathrm{P}=0.677)$ only in the "TAS \& T" pair (Table 4).

This leads to the idea that macrophages in stroma may be functionally linked to those in the tumor area, while the macrophages in the invasive front express a different functional behavior than the other two compartments.

Table III: Statistical analysis for all compartment pairs for patients with node metastasis. TAS Tumor Associated Stroma; T Tumor aria (parenchyma); IF Invasive Front.

\begin{tabular}{|l|l|l|l|l|}
\hline \multirow{2}{*}{ Compartment pairs } & \multirow{2}{*}{$\mathbf{n}$} & \multicolumn{2}{|c|}{ Correlations } & Paired Differences \\
\cline { 3 - 5 } & & Pearson correlation (R) & $\mathbf{P}$ & P \\
\hline TAS \& T pair & 40 & 0.620 & $<0.001$ & 0.061 \\
\hline TAS \& IF pair & 40 & 0.235 & 0.144 & 0.459 \\
\hline T \& IF pair & 40 & 0.498 & 0.001 & 0.391 \\
\hline
\end{tabular}

Table IV: Statistical analysis for all compartment pairs for all patients. TAS Tumor Associated Stroma; T Tumor aria (parenchyma); IF Invasive Front.

\begin{tabular}{|l|l|l|l|l|}
\hline \multirow{2}{*}{ Compartment pairs } & \multirow{2}{*}{$\mathbf{n}$} & \multicolumn{2}{|c|}{ Correlations } & Paired Differences \\
\cline { 3 - 6 } & & Pearson correlation (R) & $\mathbf{P}$ & P \\
\hline TAS \& T pair & 50 & 0.677 & $<0.001$ & 0.020 \\
\hline TAS \& IF pair & 50 & 0.368 & 0.009 & 0.341 \\
\hline T \& IF pair & 50 & 0.559 & $<0.001$ & 0.299 \\
\hline
\end{tabular}

\section{Discussions}

Regarding human breast cancers, most of the previously published studies $[19,23,24]$ demonstrate the main infiltrative tumor associated macrophages (TAM) role in tumor evolution and patient prognosis. Macrophage precursors are being recruited to and activated in the tumor site in breast cancer, as demonstrated by some exhaustive reviews $[19,23,24]$. Monocytes are being attracted to the tumor site by various chemokines as IL10 and TGF $\beta$ [19]. Previous findings on CD68 macrophage density in human breast tumor areas are contradictory and probably depend on the inconstant assessment methods or on various markers used. Leek et al [25] or Lee et al. [26] reported a diffuse infiltration pattern for TAM with no detail regarding their distribution in different tumor areas. Various statistical methods, as multivariate and univariate analysis, were applied for defining a connection between CD68 macrophages infiltration and prognosis but the results were contradictory $[2,19]$.

The present study is assessing TAM distribution by an original method of quantitative relative stained area measurement, dividing the tumor area in three regions of interest: tumor associated stroma, tumor parenchyma and invasive front. Furthermore, comparisons 
were performed among metastatic $(\mathrm{pN}>0)$ and non-metastatic ( $\mathrm{pN} 0)$ breast tumors. Even if our study was performed on only 50 cases compared to 1322 [19] or 101 [2] and a panmarker was used to stain macrophages, the information obtained are consistent with some authors [2,14] and contradicting with others [19]. Medrek [14] observed in a study on 144 cases that CD68 macrophage infiltration was present in $17 \%$ cases in the tumor parenchima and only $6 \%$ in tumor stroma.

Our study introduces standardized scanning procedures as well as contextual compartment specific analysis using predefined rules and visual validation methods. Measurements in defined tumor associated stroma, tumor parenchyma, invasive front showed variations in local CD68 macrophage density. Not only is the cell density correlated with tumor degree and partially with the prognosis but apparently also macrophage distribution in tumor areas. Our results are consistent in metastatic and non-metastatic groups and show higher CD68 TAM density in tumor site over the invasive front and tumor stroma. At the same time, in metastatic $(\mathrm{N})$ group we have observed a three time increase of CD68 macrophages in tumor areas compared to the nonmetastatic (N0) group, probably due to tumor necrosis and local hypoxia. The same pattern was found in the invasive front while in the tumor stroma, the increase of CD68 macrophage density was only two times in $\mathrm{N}$ compared to N0. Thus, regarding consistent CD68 macrophages density variations in different tumor areas, we underline the importance of assessing TAMs distribution in metastatic and nonmetastatic breast cancers.

High correlation between all three compartments found in N0 group may suggest that the macrophage play the same functional role. In the group of node metastatic patients, the fact that the invasive front had a very weak correlation with the other two compartments may indicate that many macrophages could play a different role in tumor and stroma, as opposed to invasive front, which is associated with epithelial-mesenchymal transition. Further studies which use simultaneously additional markers such as CD163 (M2-proangiogenic macrophages) and CD105 (tumor angiogenesis) seem to be necessary and could reveal more insight on the role of macrophages in breast invasive carcinoma.

\section{Conclusions}

Traceability and observer independence were improved by whole slide scanning and virtual annotation of the tumor compartments. The proposed method of relative stained area index for each of the tumor domains (tumor associated stroma, tumor parenchyma, invasive front) showed relevant differences, with higher values for tumor and invasive front, in contrast with lower values found in tumor associated stroma. When looking at groups of patients based on presence of metastasis, lymph node-negative patients revealed 3 times less CD68 stained area in both tumor and invasive front compartments. In N0 group, the high correlation between all three compartments may suggest that the macrophage play the same functional role. In contrast, in the group of node metastatic patients, the invasive front had a very weak correlation with the other two compartments indicating that macrophages could play a different role in tumor and stroma, as opposed to invasive front. The proposed scoring procedure provides a more precise measurement tool with a significant impact for patient management and eventually treatment individualization, once macrophage role is better understood. Pathologist experience in delineating tumor compartments according to morphological heterogeneity combined with our method of visually validated quantification reveal new perspectives on macrophage role in invasive breast carcinoma, for clinical diagnosis.

\section{Conflict of Interests}

Authors have no conflict of interests to declare.

\section{Aknowledgements}

The first author is Ph.D. student at "Gr.T. Popa" University of Medicine and Pharmacy, Iaşi, Romania. This paper is the result of the work undertaken during the doctoral internship.

\section{References}

1. Coussens LM, Werb Z (2002) Inflammation and cancer. Nature 420 860-867.

2. Leek RD, Harris AL (2002) Tumor-associated macrophages in breast cancer. J Mammary Gland Biol Neoplasia 7: 177-189.

3. Mantovani A, Sozzani S, Locati M, Allavena P, Sica A (2002) Macrophage polarization: tumor-associated macrophages as a paradigm for polarized M2 mononuclear phagocytes. Trends Immunol 23: 549-555.

4. Mantovani A, Bottazzi B, Colotta F, Sozzani S, Ruco L (1992) The origin and function of tumor-associated macrophages. Immunol Today 13: 265-270.

5. Bingle L, Brown NJ, Lewis CE (2002) The role of tumour-associated macrophages in tumour progression: implications for new anticancer therapies. J Pathol 196: 254-265.

6. Allavena P, Mantovani A. Immunology in the clinic review series; focus on cancer: tumour-associated macrophages: undisputed stars of the inflammatory tumour microenvironment. Clin Exp Immunol. 2012; 167(2): 195-205.

7. Ostrand-Rosenberg S (2008) Immune surveillance: a balance between protumor and antitumor immunity. Curr Opin Genet Dev 18: 11-18.

8. Dalton DK, Pitts-Meek S, Keshav S, Figari IS, Bradley A, et al. (1993) Multiple defects of immune cell function in mice with disrupted interferon-gamma genes. Science 259: 1739-1742.

9. Gordon S (2003) Alternative activation of macrophages. Nat Rev Immunol 3: 23-35.

10. Tang X (2013) Tumor-associated macrophages as potential diagnostic and prognostic biomarkers in breast cancer. Cancer Lett 332: 3-10.

11. Alkhateeb AA, Han B, Connor JR. Ferritin stimulates breast cancer cells through an iron-independent mechanism and is localized within tumorassociated macrophages. Breast Cancer Res Treat. 2013; 137(3): 733-744.

12. Ch'ng ES, Tuan Sharif SE, Jaafar H. In human invasive breast ductal carcinoma, tumor stromal macrophages and tumor nest macrophages have distinct relationships with clinicopathological parameters and tumor angiogenesis. Virchows Arch. 2013; 462(3): 257-267.

13. Heys SD, Stewart KN, McKenzie EJ, Miller ID, Wong SY, et al. (2012) Characterisation of tumour-infiltrating macrophages: impact on response and survival in patients receiving primary chemotherapy for breast cancer. Breast Cancer Res Treat 135: 539-548.

14. Medrek C, Pontén F, Jirström K, Leandersson K (2012) The presence of tumor associated macrophages in tumor stroma as a prognostic marker for breast cancer patients. BMC Cancer 12: 306.

15. Mantovani A, Allavena P, Sica A, Balkwill F (2008) Cancer-related inflammation. Nature 454: 436-444.

16. Mantovani A, Marchesi F, Porta C, Sica A, Allavena P (2007) Inflammation and cancer: breast cancer as a prototype. Breast 16 Suppl 2: S27-33.

17. Balkwill F, Mantovani A (2001) Inflammation and cancer: back to Virchow? Lancet 357: 539-545. 
Citation: Haisan A, Petreus T, Jitaru D, Danciu M ,Carasevici E. Macrophage Quantification in Different Breast Tumor Compartments. Journal of Surgery [Jurnalul de chirurgie] 2014; 10(1): 43-48. doi: 10.7438/1584-9341-10-1-8

18. Mahmoud SM, Paish EC, Powe DG, Macmillan RD, Grainge MJ, et al (2011) Tumor-infiltrating CD8+ lymphocytes predict clinical outcome in breast cancer. J Clin Oncol 29: 1949-1955.

19. Mahmoud SM, Lee AH, Paish EC, Macmillan RD, Ellis IO, et al. (2012) Tumour-infiltrating macrophages and clinical outcome in breast cancer. J Clin Pathol 65: 159-163.

20. Campbell MJ, Tonlaar NY, Garwood ER, Huo D, Moore DH, et al. (2011) Proliferating macrophages associated with high grade, hormone receptor negative breast cancer and poor clinical outcome. Breast Cancer Res Treat 128: 703-711.

21. Fan C, Oh DS, Wessels L, Weigelt B, Nuyten DS, et al. (2006) Concordance among gene-expression-based predictors for breast cancer. N Engl J Med 355: 560-569.

22. Shih JY, Yuan A, Chen JJ-W, Yang PC. Tumor-Associated Macrophage: Its Role in Cancer Invasion and Metastasis. J Cancer Mol. 2006; 2: 101-106.
23. Ch'ng ES, Jaafar H, Tuan Sharif SE (2011) Breast Tumor Angiogenesis and Tumor-Associated Macrophages: Histopathologist's Perspective. Patholog Res Int 2011: 572706.

24. Mukhtar RA, Nseyo O, Campbell MJ, Esserman LJ (2011) Tumorassociated macrophages in breast cancer as potential biomarkers for new treatments and diagnostics. Expert Rev Mol Diagn 11: 91-100.

25. Leek RD, Lewis CE, Whitehouse R, Greenall M, Clarke J, et al. (1996) Association of macrophage infiltration with angiogenesis and prognosis in invasive breast carcinoma. Cancer Res 56: 4625-4629.

26. Lee AH, Happerfield LC, Bobrow LG, Millis RR (1997) Angiogenesis and inflammation in invasive carcinoma of the breast. J Clin Pathol 50: 669-673.

27. Ueno H, Jones AM, Wilkinson KH, Jass JR, Talbot IC (2004) Histological categorisation of fibrotic cancer stroma in advanced rectal cancer. Gut 53: 581-586. 